\title{
Non-medical prescribing of parenteral nutrition
}

\author{
S. Harwood, A. Jukes, W. Magambo, S. Tracey and A. B. Hawthorne \\ University Hospital of Wales, Heath Park, Cardiff CF14 4XW, UK
}

The prescribing of parenteral nutrition (PN) was identified as a problem in 2005 following an in house audit. In many cases PN was not being prescribed at all, in others it was being prescribed incorrectly. This had profound legal implications as PN, which is a prescription only medicine, was being compounded, supplied and administered where no legal prescription existed.

Having identified a need to improve the prescribing of PN, the nutrition support team (NST) pharmacist secured funding to undertake the non-medical prescribing course. In order to complete the course, the pharmacist gained support from both the lead clinician for the NST, her pharmaceutical and NST colleagues.

A 'PN prescription details' chart has been developed detailing the content of the PN.

A programme of education and training is being carried out by the NST where the legal classification of PN, the need for a written prescription and the role of non-medical prescribing is highlighted.

The pharmacist qualified as a non-medical prescriber in March 2008. Since then a clinical management plan has been developed for inpatients receiving PN. Although obtaining patient consent is not a problem with patients who are alert and mentally well, it causes difficulties in patients who are unwell. In these cases, consent is obtained at the earliest possible opportunity and in the meantime, working with the medical team overseeing the care of the patient, the PN is prescribed by the non-medical prescriber. In obtaining patient consent, the NST have found themselves undertaking a more active role in explaining to the patient the processes involved in receiving PN

Local audits have shown vast improvements in the prescribing of PN with almost $100 \%$ of PN now being prescribed correctly.

A guideline in conjunction with the 'PN prescription details' chart has been submitted to the Cardiff and Vale NHS Trust for ratification and which may then be used by others in the absence of the non-medical prescriber.

Written information is being developed to give to patients to explain the nature of non-medical prescribing and to be used as an aid to obtain patient consent.

The prescribing of PN is now consistent and accurate following the implementation of non-medical prescribing. Having implemented this process patient care and record keeping has been improved. 